\title{
Characteristics of vibrations in domestic environments as sources for kinetic energy harvesters
}

\author{
Mario Mösch, Gerhard Fischerauer \\ Chair of Measurement and Control Systems and Center of Energy Technology (ZET), \\ Universität Bayreuth, 95440 Bayreuth, Germany \\ mrt@uni-bayreuth.de
}

\begin{abstract}
Kinetic energy harvesters convert vibration energy to usable electrical energy. They work best when adapted to their environment. In particular, the resonance frequency of a resonant harvester should match the vibration frequency; otherwise, the harvested power decreases sharply. It is therefore important to know the vibration characteristics of the environment as well as possible. This work concerns itself with vibrations in domestic environments such as passive vibrations of solid structures like walls and ceilings or active vibrations of household devices. Particular attention is given to timevariant vibrations, i. e., to vibrations the characteristics of which vary with time. The results lead to guidelines for the mounting of kinetic energy harvesters and for useful harvester designs. We also present a sub-10- $\mu \mathrm{W}$ wireless sensor node for domestic temperature measurement. The small power consumption means that vibrations with small acceleration amplitudes suffice to power it via kinetic energy harvesting.
\end{abstract}

Keywords: Energy harvesting, vibration sources, characteristics, domestic environments, wireless sensor nodes.

\section{Introduction}

Wireless sensor networks (WSN) are advertised as basis for flexible condition monitoring or home automation tasks [1]. To fully exploit their potential, the WSN nodes must be operated cableless and without batteries. A potential approach to solve this problem is energy harvesting. Kinetic energy harvesters convert energy from the mechanical to the electrical domain by, e. g., electromagnetic [2] or piezoelectric principles [3]. The resonance frequency of a resonant harvester should match the vibration frequency; otherwise, the harvested power decreases sharply and may not suffice to power a WSN node.

Several solutions for this issue have been investigated in the past. They can be categorized according to their strategy. The first strategy is to widen the harvester's bandwidth, e.g., by building arrays with various resonance frequencies [4] or by nonlinear systems [5]. The second strategy is to change the resonance frequency with an adaptation mechanism, which can increase the output power unless the adaption process consumes too much energy itself [6], [7].

In either case, it is crucial to know the characteristics of the environmental vibrations so that a harvester with optimum response to these characteristics can be designed. The open literature reports on at least partial vibration measurements. Ref. [8] lists the resonance frequency and the acceleration amplitude of various sources such as a car hood, a microwave oven, and a tree. Ref. [9] does the same with household devices, e.g., a freezer and a washing machine, but does not discuss the influence of different operating points (device settings). Ref. [10] treats similar vibration sources as [8] and also goes into the details of time-variant spectra.

In our work, we characterized vibrations in domestic environments in an effort to derive rules for the design and the mounting of kinetic energy harvesters. We did not only consider the strengths and frequencies of the vibrations, but also determined how the vibration characteristics change as a function of time and the operating points of the respective vibration sources.

From this, consequences for vibration energy harvesting have been derived. We also demonstrate with an ultra-low-power WSN node how low-acceleration vibration sources can be exploited to power kinetic energy harvesters. 


\section{Minimum usable acceleration amplitude}

We used acceleration measurements to characterize vibration sources. Assuming a puremode (monofrequent) vibration for simplicity's sake, the minimum acceleration amplitude required to power a WSN node is estimated as follows.

First note that the available electrical power $P_{\mathrm{el}}$ of a kinetic energy harvester at resonance is

$$
P_{\text {el }}=m^{2} A^{2} c_{\text {load }} / 2 c_{\mathrm{t}}^{2}
$$

with the mass $m$, the acceleration amplitude $A$, and the load and total damping coefficients $c_{\text {load }}$ and $c_{\mathrm{t}}$ [11]. The prototype of Ref. [2] harvests $46 \mu \mathrm{W}$ of power with $m=0.66 \mathrm{~g}$ and a vibration with amplitude $A=0.59 \mathrm{~ms}^{-2}$ at $f=52 \mathrm{~Hz}$. This prototype would harvest $10 \mu \mathrm{W}$ with $m=1.82 \mathrm{~g}$ and $A=0.1 \mathrm{~ms}^{-2}$ and otherwise unchanged conditions (the effective spring stiffness $k$ needs to be modified to keep the resonant frequency $f_{r}=\sqrt{k / m} /(2 \pi)$ constant). This value of $10 \mu \mathrm{W}$ suffices to power a WSN node as is demonstrated below.

This leads us to believe that an acceleration amplitude of $A_{\min }=0.1 \mathrm{~ms}^{-2}$ is a reasonable estimate for the minimum vibration strength required to permanently supply a WSN node with its power.

(a)

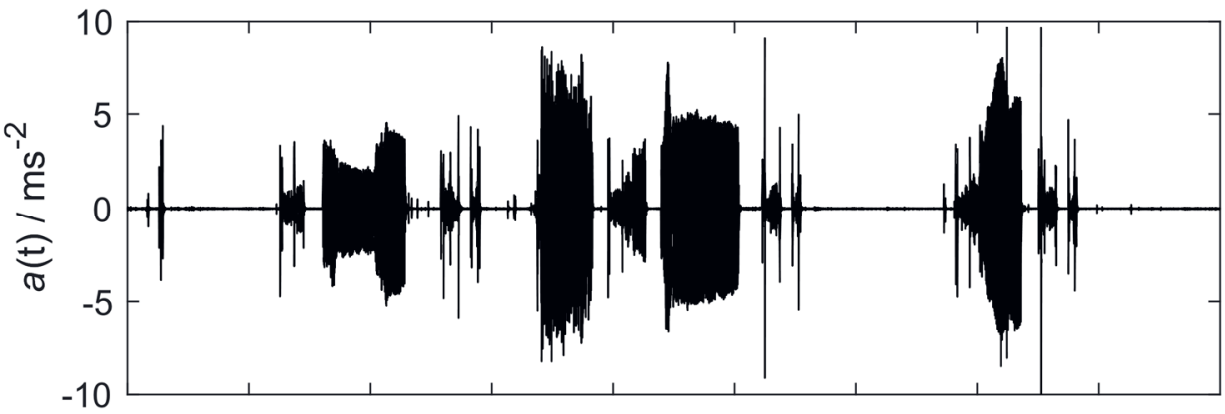

(b)

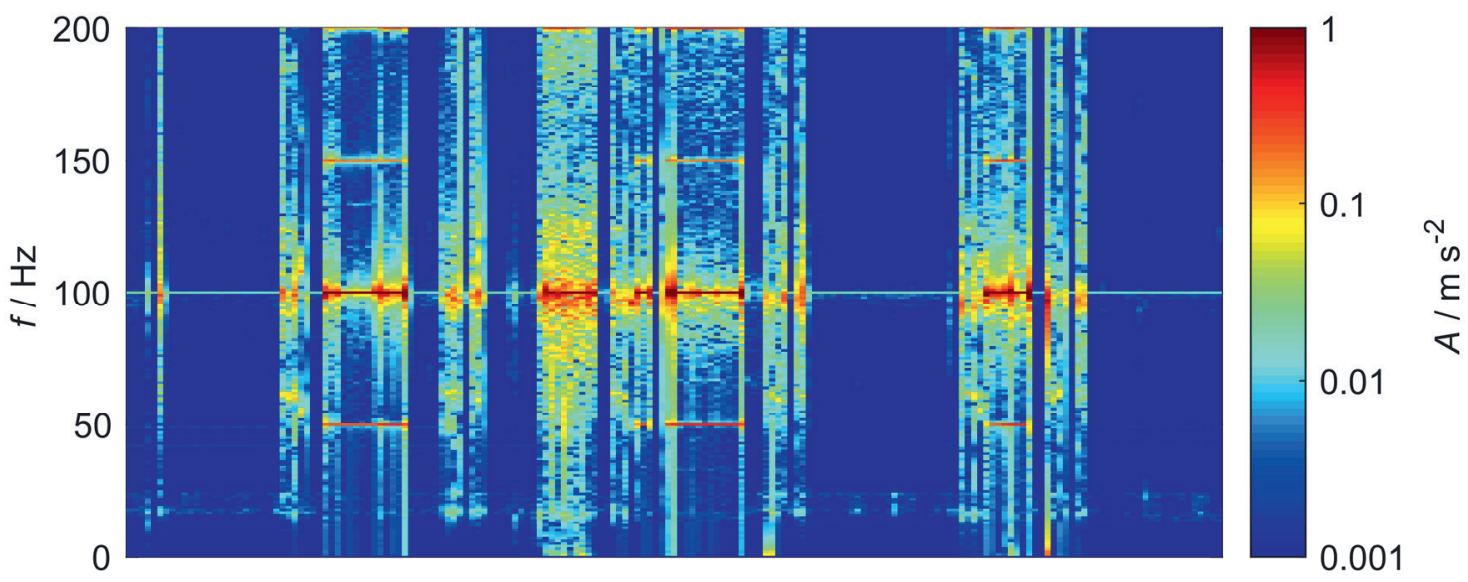

(c)

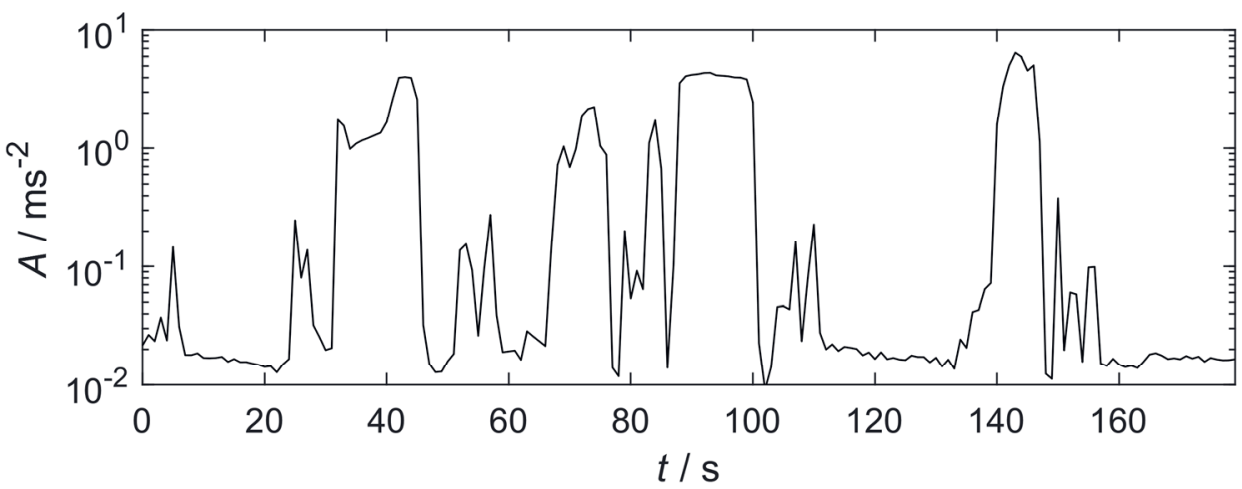

Fig. 1: Vibration of an automatic coffee machine during brewing. (a) Measured time response. (b) Spectrogram by short-time Fourier-transformation. (c) Time response of the 100-Hz vibration component. (See text on next page.) 


\section{Measurement approach}

We investigated the vibrations on the floor, the ceiling, and the walls of a detached house and an office building and on devices operated therein, among them a microwave oven, a refrigerator, a kitchen hood, and an oil heating.

For the measurement, we mounted a highsensitive acceleration sensor (PCB 356B18) on the vibration source to be investigated. Its output signal was amplified and registered by mobile measurement equipment (PCB480B21, $\mathrm{NI}$-9239). The acceleration time series was transformed to the frequency domain by Fourier transformation. To cope with timevariant signals, we used the short-time Fourier transform (STFT).

By way of an example, Fig. 1 on the preceding page shows the results for an automatic coffee machine during brewing. One notes the timevariance of the vibration.

\section{Measurement results}

Figure 2 shows the amplitude spectra of vibrations on the floor, on a window frame, and on a door frame in the same room of a detached house. A resonance at $49.5 \mathrm{~Hz}$, which is no mains interference, is present in every spectrum, while additional resonances at 24, 39, and $90.4 \mathrm{~Hz}$ occur only occasionally. The acceleration amplitudes of less than $3 \cdot 10^{-4} \mathrm{~ms}^{-2}$ are far below $A_{\min }$. Such results are typical for passive vibrations of solid structures or switched-off devices.

More promising energy harvesting sources are shock vibrations. They occur nearly everywhere with peak accelerations up to several tens of $\mathrm{ms}^{-2}$ in domestic environments. The strength depends on the exact source of the shock, especially when created by human interaction, and the distance from the source. Furthermore, the typical duration of shock vibrations is in the seconds range or below.

Figure 3 shows an example, a 200-ms shock vibration with a peak acceleration amplitude of $0.5 \mathrm{~ms}^{-2}$. The corresponding amplitude spectrum reveals that the dominant frequencies are about $100 \mathrm{~Hz}$ and $160 \mathrm{~Hz}$, but the total spectrum is rather broadband. Such a broadband and high-peak vibration calls for special harvester geometries, e. g., piezoelectric membranes [12].

A drawback of shock vibrations is, of course, that they result from events that are difficult to predict. Consequently, shock vibrations are not suitable as energy sources for continuously operated WSN nodes, but can be used to trigger event-driven actions.
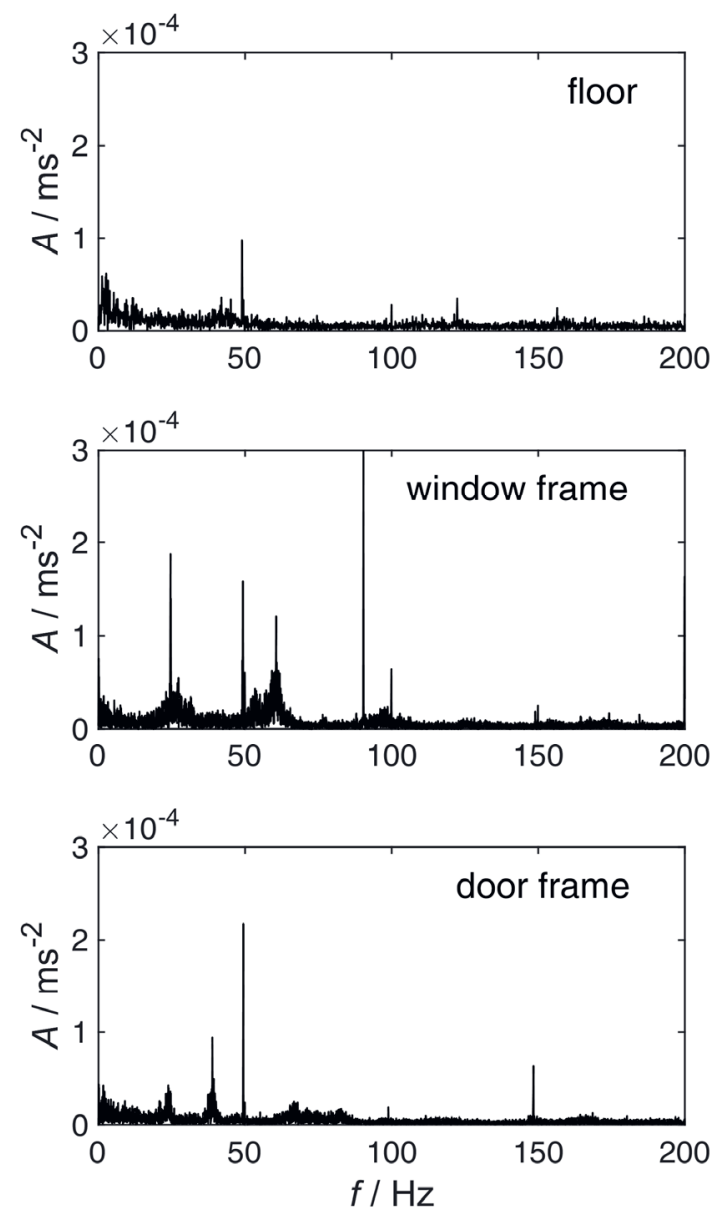

Fig. 2: Typical amplitude spectra of solidstructure vibrations in domestic environments. The resonances are too weak to be of practical use.
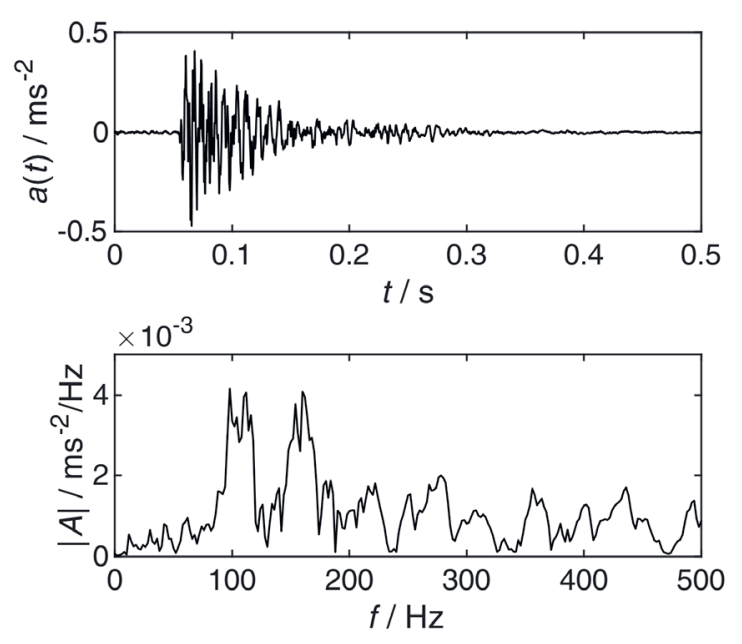

Fig. 3: Time response and associated amplitude spectrum of a shock vibration.

Household devices as potential vibration sources can be classified into devices with moving inner parts (e. g., washing machines) and other devices (e. g., TV sets). The latter are in the 

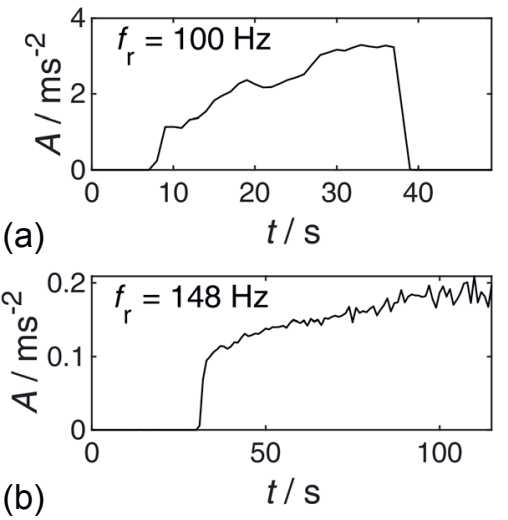

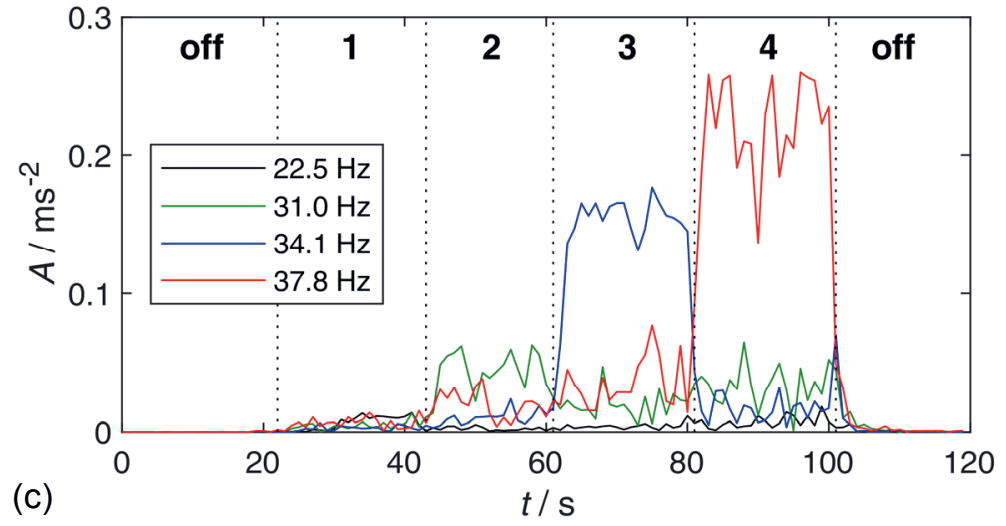

Fig. 4: Measured characteristics of some household devices. (a) Time-variant acceleration amplitude $A$ at the main resonance frequency for a microwave oven. (b) As (a), but for a refrigerator. (c) Acceleration amplitudes at various frequencies for a kitchen hood over time. Different power stages (off, 1-4) obviously result in different main resonances. 1: $22.5 \mathrm{~Hz}$; 2: $31.0 \mathrm{~Hz}$; 3: $34.1 \mathrm{~Hz}$; $4: 37.8 \mathrm{~Hz}$ ).

same category as solid structures and switched-off devices. It is self-evident that only active vibrating devices are potential energy sources.

Based on their characteristics, these vibrating household devices can be subdivided into two classes V1, V2. The first class V1 comprises devices with a constant dominant resonance frequency. The coffee machine described by the results shown in Figure 1 belongs to this group of devices, even though the amplitude spectrum is broadened every so often because of the coffee grinding process. The maximum acceleration amplitude of the $100-\mathrm{Hz}$ mode exceeds the $1 \mathrm{~ms}^{-2}$ limit several times and can reach up to $4.1 \mathrm{~ms}^{-2}$. At other times, the acceleration amplitude stays below $A_{\text {min }}$.

Microwave ovens and refrigerators share similar characteristics with coffee machines in that their vibrations are dominated by singlemode resonances, cf. Figs. 4(a) and (b). The dominant $100-\mathrm{Hz}$ mode of the microwave oven in Fig. 4(a) vibrates with acceleration amplitudes between 1 and $3 \mathrm{~ms}^{-2}$ whereas the 148$\mathrm{Hz}$ mode of the refrigerator in Fig. 4(b) vibrates with amplitudes between 0.1 and $0.2 \mathrm{~ms}^{-2}$. The time-variance of the vibration amplitudes has to be taken into account for the harvester design for two reasons. First, the maximum geometrical deflection of the harvester is a design parameter. Second, the expected harvested power influences how the overall system is to operated, e. g., how often a WSN node can transmit data.

The second class V2 consists of devices with variable-speed drives. Higher rotation speeds amount to higher resonance frequencies. Such effects are observed, e.g., with washing machines and kitchen hoods.
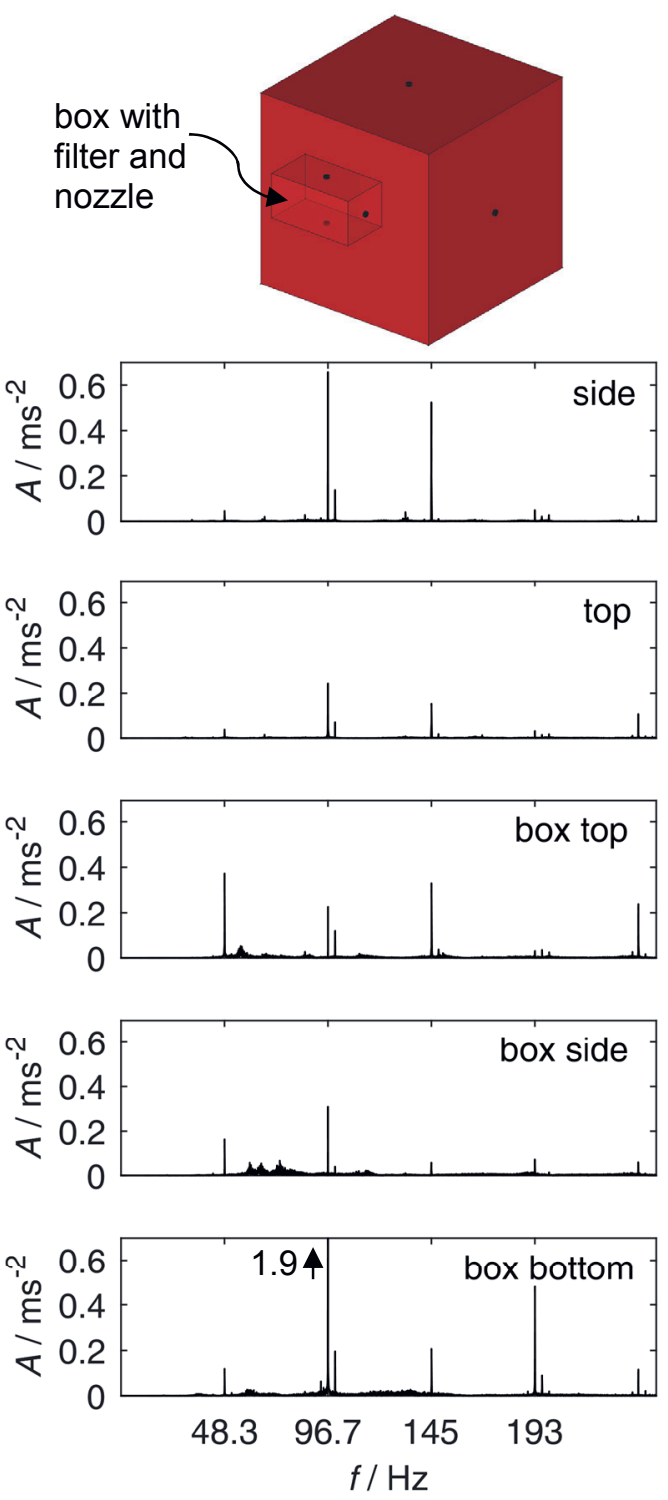

Fig. 5: Amplitude spectra of the vibrations on the surface of an oil heating. 


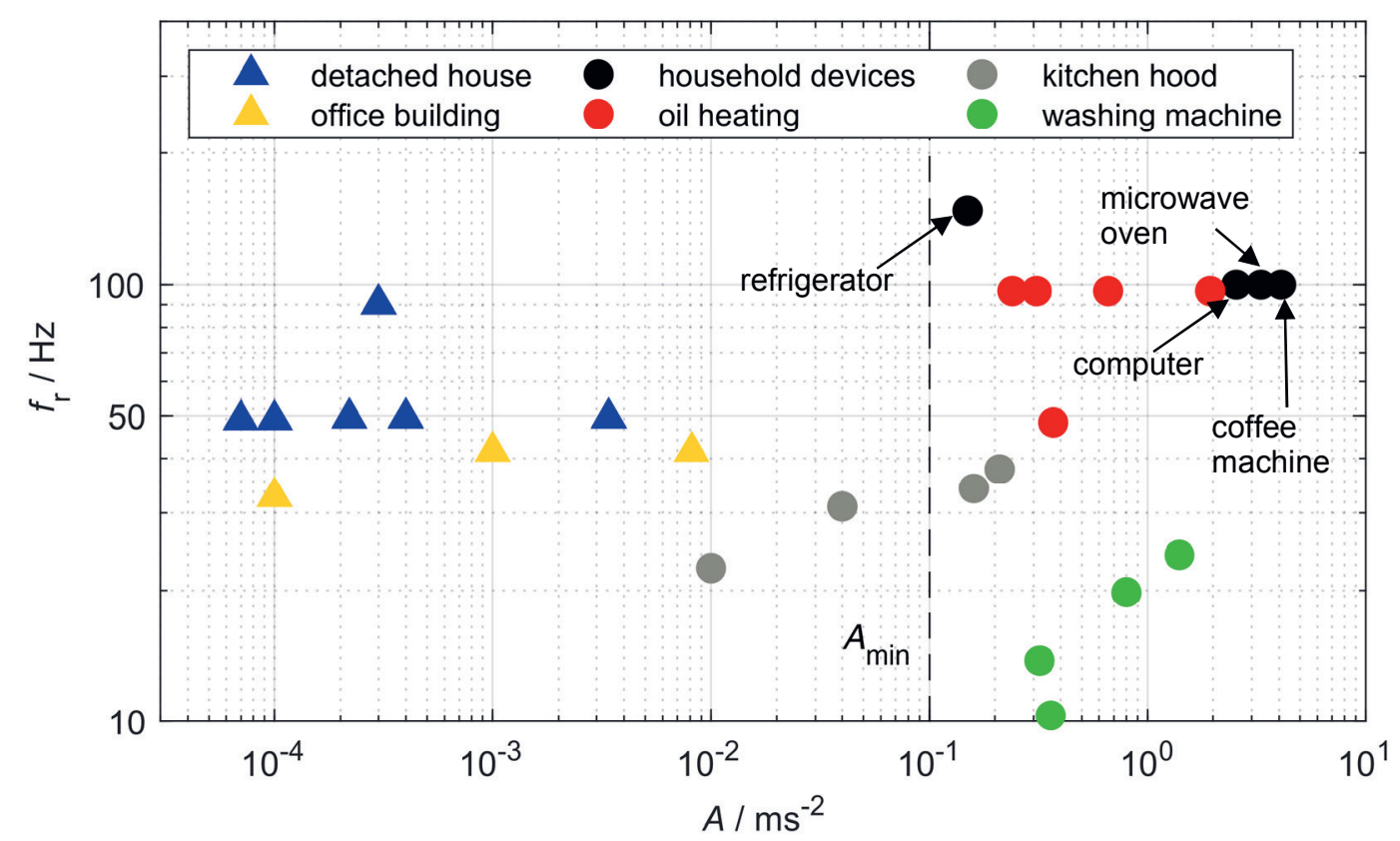

Fig. 6: Dominant resonance frequencies and associated acceleration amplitudes $A$ of domestic vibration sources. Triangles and circles respectively mark passively and actively vibrating sources.

A washing machine tested by us offered various programs with four different speeds $(600,800,1200$, and $1600 \mathrm{rpm})$. The associated dominant resonance frequencies were about 10, 13, 20, and $27 \mathrm{~Hz}$. The acceleration amplitudes varied from 0.3 to $1.4 \mathrm{~ms}^{-2}$. An additional $100-\mathrm{Hz}$ vibration could be tracked to the water pump and had amplitudes between 0.1 and $0.4 \mathrm{~ms}^{-2}$.

A tested kitchen hood had four operating points with increasing suction due to increasing rotational speed of the fan motor. This leads to time-variant dominant vibration frequencies and amplitudes, cf. Fig. 4(c). It goes without saying that such time-variant behavior requires adaptive energy harvesters if the kinetic energy is to be harvested with maximum efficiency at each operating point.

The influence of the mounting position of an energy harvester was studied on the basis of an oil heating located in the basement of a detached house. The results are visualized in Fig. 5 on the preceding page. One observes a fundamental resonance at $48.3 \mathrm{~Hz}$ and its harmonics. Depending on the position of the acceleration sensor, quite different amplitudes were measured. The use of the $96.7-\mathrm{Hz}$ signal appears to be the best overall choice as this signal is always present and is always above $A_{\min }$. The general lesson from this is that the mounting position of an energy harvester on a vibration source well-suited in principle actually determines whether the results will be useful or useless.

\section{Consequences for energy harvesting}

Figure 6 shows the observed frequencies and acceleration amplitudes of the strongest vibrations in various cases. The acceleration amplitudes cover about five orders of magnitude. As a general rule, the low amplitudes occur for passively vibrating sources such as walls, ceilings, and floors, whereas the higher amplitudes above the useful limit of $A_{\min }=$ $0.1 \mathrm{~m} / \mathrm{s}^{2}$ involve actively vibrating sources. The highest acceleration amplitude $\left(4 \mathrm{~m} / \mathrm{s}^{2}\right)$ was measured on the surface of a coffee machine.

Most resonances of actively vibrating devices in the household provide strong enough amplitudes to power WSN nodes. The resonances usually occur at the mains frequency of $50 \mathrm{~Hz}$ or multiples thereof. In these cases, linear vibration energy harvesters with fixed resonance frequencies of 50 or $100 \mathrm{~Hz}$ are sufficient. Only time-variant sources with variable-speed drives such as washing machines or kitchen hoods call for self-adapting harvesters [7].

\section{Ultra-low-power WSN node for temperature measurement}

We have assumed above that an acceleration amplitude of $A_{\min }=0.1 \mathrm{~m} / \mathrm{s}^{2}$, which leads to a harvested power of $10 \mu \mathrm{W}$, may suffice for practical applications. This needs to be demonstrated. To this end, we considered a WSN node for home automation which measures the air temperature and transmits the result to a base station. 

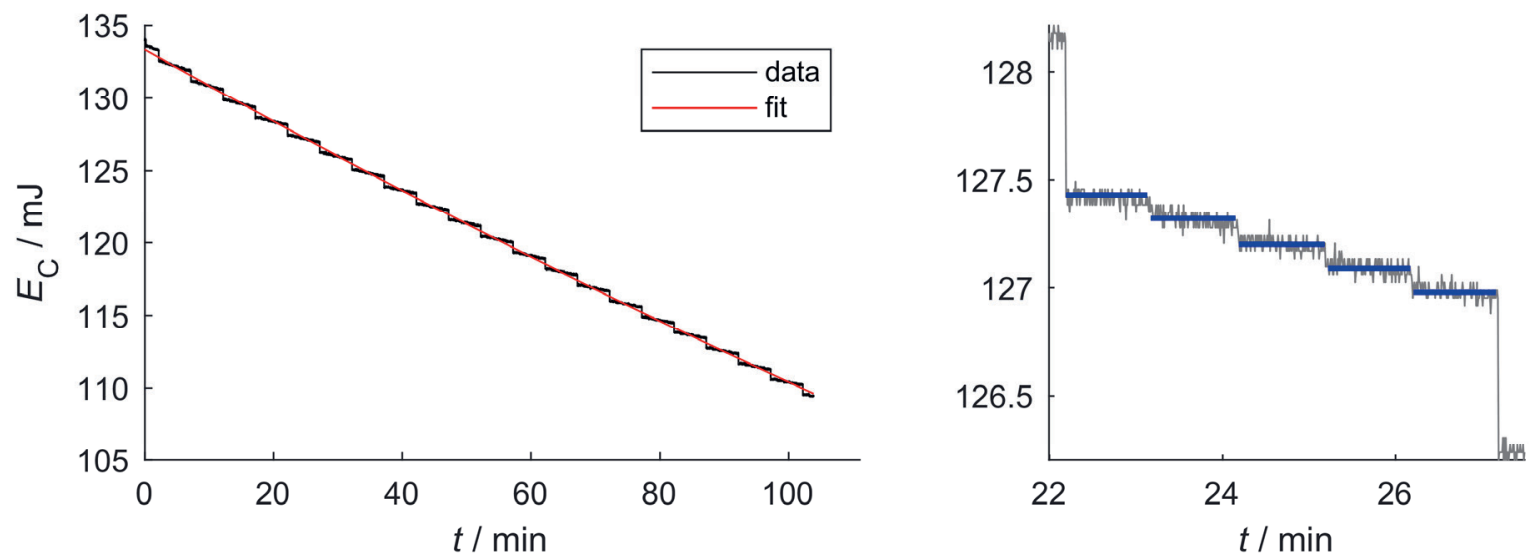

Fig. 7: Energy stored by a 47-mF capacitor connected to a WSN node with temperature sensor. The node transmits measurement values at intervals of $T_{T x}=300 \mathrm{~s}$. (a) Long-term measurement ( - ) and quadratic fit (-). (b) Detail of (a). The sensor averages $N=5$ measured temperatures to obtain the final result. The horizontal blue lines mark deep-sleep periods.

To reduce the average power consumption of the WSN node, it is operated with alternating short measurement and data transmission cycles and long low-power (sleep) cycles. The WSN node consisted of a microcontroller (MCU) on a development board (STM32L452), a radio-frequency (RF) frontend for communication in the ISM band at $868 \mathrm{MHz}$ (XNUCLEO-IDS01A4) and an acceleration sensor with built-in temperature measurement capability (ADXL362). The MCU communicates with the RF frontend and the sensor via SPI bus.

After the cyclic wake-up from the deep-sleep state, the MCU initializes the sensor and receives several temperature values. The MCU enters a medium-sleep mode when waiting for a new value to save energy and is wakened by a pin interrupt. After having received several temperature values, the average temperature is transmitted to the basestation by RF communication at a transmit power of $-10 \mathrm{dBm}$. Afterwards, the whole sensor node enters the deep-sleep mode with everything switched off except the real-time clock. The latter acts as an alarm clock which periodically wakes up the MCU. To reduce the power consumption, we also tested an alternative scenario in which the average temperature is stored by the $\mathrm{MCu}$, but only transmitted every fifth time.

The sensor node is powered by a $47-\mathrm{mF}$ capacitor. The system is operated such that the capacitor voltage stays between 1.8 and $2.8 \mathrm{~V}$. Fig. 7 shows an example of the measured energy stored by the capacitor as a function of time. The instances at which the energy drops coincide with temperature measurement and data transmission processes.

\begin{tabular}{lll}
\hline$T_{\mathrm{TX}} / \mathrm{s}$ & $N$ & $P_{\mathrm{av}} / \mu \mathrm{W}$ \\
\hline 10 & 1 & 67 \\
60 & 1 & 11.5 \\
300 & $1 / 5$ & $2.9 / 3.6$ \\
\hline
\end{tabular}

Table 1: Average power $P_{a v}$ required by the WSN node at a capacitor voltage of $2.2 \mathrm{~V}$. The node transmits $N$ averaged temperature values at time intervals of $T_{T x}$ (in other words, the deep-sleep time between two successive measurements is $T_{T_{x}} / N$ ).

Other operation scenarios than the one shown in Fig. 7 have been investigated. Since the power consumption of the MCU depends on its supply voltage, we aimed at making the results comparable by converting all measured capacitor energies to the values they would have had at a voltage of $2.2 \mathrm{~V}$. This was done by quadratic fit functions such as the one shown in Fig. 7.

The influence of various operation scenarios on the WSN node power consumption is detailed in Table 1. Data transmission every $60 \mathrm{~s}$ and every $300 \mathrm{~s}$ respectively leads to an average power consumption of $11.5 \mu \mathrm{W}$ and 3 to $4 \mu \mathrm{W}$. This justifies our prior assumption of a useful acceleration amplitude limit of $A_{\min }=$ $0.1 \mathrm{~m} / \mathrm{s}^{2}$.

\section{Conclusions}

We have experimentally characterized potential sources for vibration energy harvesting in domestic environments. It turns out that passively vibrating solid structures cannot power a WSN node via vibration energy harvesting. An exception are shock vibrations for event-driven systems. 
The vibrations of several household devices, on the other hand, are strong enough to be useful for energy harvesting. We have encountered both stable narrow-band resonances and time-variant broad-band vibrations, which influences the optimum harvester design and its mode of operation. We have also shown that the mounting position of an energy harvester on the surface of a vibrating device is of critical importance.

Finally, we have shown by means of a demonstrator that an acceleration amplitude of $0.1 \mathrm{~m} / \mathrm{s}^{2}$, corresponding to an harvested power of $10 \mu \mathrm{W}$, suffices to operate an ultra-lowpower WSN node.

\section{Funding}

This work was partially funded by the Bavarian State Ministry of Education, Science and the Arts within the framework of TechnologieAllianzOberfranken (TAO).

\section{References}

[1] M. Li, H.-J. Lin, IEEE Trans. Indust. Electron. 62, 4430-4442 (2015); doi: 10.1109/TIE.2014. 2379586.

[2] S. P. Beeby et al., J. Micromech. Microeng. 17, 1257-65 (2007); doi: 10.1088/0960-1317/17/7/ 007.

[3] A. Khalid, A. K. Redhewal, Mat. Sci. Appl. 6, 818-827 (2015); doi: 10.4236/msa.2015. 69084.

[4] D. Zhu, M.J. Tudor, S. P. Beeby, Meas. Sci. Technol. 21, 022001 (2010); doi: 10.1088/ 0957-0233/21/2/022001.

[5] M. F. Daqaq et al., Appl. Mech. Rev. 66, 040801 (2014); doi: 10.1115/1.4026278.

[6] D. Hoffmann et al., Smart Mater. Struct. 25, 035013 (2016); doi: 10.1088/0964-1726/25/3/ 035013.

[7] M. Mösch, G. Fischerauer, Micromachines 10, 44 (2019); doi: 10.3390/mi10010044.

[8] N. E. duToit, B. L. Wardle, S.-G. Kim, Integr. Ferroelectr. 71, 121-160 (2005); doi: 10.1080/ 10584580590964574.

[9] J.W. Matiko et al., Meas. Sci. Technol. 25, 012002 (2014); doi: 10.1088/0957-0233/25/1/ 012002.

[10] S. P. Beeby et al., Smart Mater. Struct. 22, 075022 (2013); doi: 10.1088/0964-726/22/7/ 075022.

[11] N. G. Stephen, J. Sound Vibr. 293, 409-25 (2006); doi: 10.1016/j.jsv.2005.10.003.

[12] D. Alghisi et al., Sensors Actuat. A 233, 569-81 (2015); doi: 10.1016/j.sna.2015.07.020. 